\title{
Les pratiques sexuelles phallocentriques chez Arcan : quelques procédés littéraires de désérotisation
}

Christina BRASSARD, Université de Toronto

\section{Le culte du phallus}

En Occident, depuis l'Antiquité, le phallus - représentation du membre viril - est un symbole imposant dans les sociétés patriarcales. Symbole sacré, symbole initiatique, symbole de puissance et de virilité, le phallus a souvent eu droit à une représentation positive et glorieuse, d'autant plus que, selon 1'historien français Alain Daniélou, «nous retrouvons sa vénération à l'origine de toutes religions » (10). «[L]e phallus, constitué en symbole de la virilité » (39), comme l'affirme Pierre Bourdieu dans son ouvrage La domination masculine, s'est vu aussi devenir l'emblème par excellence de la fécondité et de la force - pensons au dieu Priape dans la mythologie grecque et à son membre viril constamment en érection. Dans les théories psychanalytiques, le phallus - qui signifie davantage que la simple représentation du membre viril - est par ailleurs le centre de tout. Chez Sigmund Freud, par exemple, le symbolisme phallique est au cœur de la structure psychique de l'individu, tandis que chez Jacques Lacan, le phallus est le signifiant même du désir sur lequel la société occidentale s'est construite. Comme le rappelle JeanJoseph Goux, « le phallus, tel que la psychanalyse de Lacan le théorise distinctement, se présente comme l'équivalent général des objets de pulsion » (10).

Le terme «phallocentrisme » désigne justement ce système de pensée qui a été activement dénoncé par les féministes, notamment par Simone de Beauvoir qui atteste la trop grande influence du pénis dans la construction identitaire du sujet masculin : «Tandis que le garçon se recherche dans le pénis en tant que sujet autonome, la fillette dorlote sa poupée et la pare comme elle rêve d'être parée et dorlotée » (25). C'est ce «phallocentrisme » qui provoquait - et provoque encore souvent - chez les hommes la peur du féminin, car, comme l'évoque Bourdieu, qui dit virilité, dit refus de la fragilité et de la féminité : «La virilité, on le voit, est une notion éminemment relationnelle, construite devant et pour les autres hommes et contre la féminité, dans une sorte de peur du féminin » (78). L'idéalisation du phallus est aussi évoquée chez Judith Butler sous le terme «phallogocentrisme »(89), qu'elle emprunte à Michel Foucault, et Joan Scott, historienne 
américaine renommée, explique quelques revers de ce «système phallique » (172) dans De l'utilité du genre.

Il est manifeste que la puissance masculine, plus précisément la « virilité », communément appelée aujourd'hui « masculinité » ${ }^{1}$ (Courtine 8), a longtemps été liée au membre viril en érection - ce membre viril qui devait, de tout son symbolisme, dominer la féminité. Les études dérivées de l'ensemble des travaux de Raewyn Connell rendent compte du fait que «le corps est incontournable dans la construction de la masculinité » (45) mais que, « [...] comme il s'agit de configurations de pratiques et non d'identités figées, [1]es modalités de la masculinité sont soumises au changement et à l'histoire ; elles peuvent se reconfigurer » (11). Le membre viril est spécifiquement l'une des parties du corps les plus sujettes à façonner l'identité masculine, car il a influencé la construction sociale de nos sociétés occidentales. On sait cependant que depuis la Seconde Guerre mondiale, la Révolution tranquille et les mouvements féministes, la société occidentale phallocentrique a peu à peu changé, et ce, sous différents angles - à titre de preuve, nous n'avons qu'à consulter les trois tomes collectifs, Histoire de la virilité 1 (Vigarello), Histoire de la virilité 2 (Corbin) et Histoire de la virilité 3 (Courtine), pour nous apercevoir rapidement que des changements ont évidemment eu lieu au fil des siècles. Dans son ouvrage intitulé Renversements : l'or, le père, le phallus et le langage, Goux note :

Il devient clair aujourd'hui [...] qu'il n'est guère d'aspect de la vie contemporaine, personnelle ou publique, qui n'ait été marquée par les idées, les projets, les innovations qui ont fait irruption à la fin des années soixante $[. .$.$] . Ce fut une époque$ de contestations et de ruptures profondes [...]. Le mouvement des femmes, la sensibilité écologique, sans parler de tout ce qui atteste d'un changement dans les rapports interpersonnels, en sont les fruits les plus incontestés. (19)

Assurément, quoique les sociétés occidentales soient en changement depuis les années 1960, comme Goux le suggère, et que l'idée de «virilité » ait évolué, comme le mettent en perspective les ouvrages collectifs sur l'histoire de la virilité, nous avons encore du travail à accomplir afin de rendre nos constructions sociales moins phallocentrées, notamment en ce qui a trait aux pratiques sexuelles.

C'est peut-être justement sur la voie du (dé)phallocentrisme que l'écriture de Nelly Arcan nous amène à plusieurs reprises. On voit nettement que la manière dont Arcan écrit sur le membre viril est bien particulière et met en lumière un certain renversement dans sa représentation comme objet de désir. En outre, en critiquant la place que détient le membre viril dans un certain type de 
pratiques sexuelles, Arcan montre que le patriarcat doit encore être contesté et modifié, et ce, pour des raisons éminemment justifiables : il a laissé des marques profondes sur notre sexualité.

\section{L'écriture arcanienne}

On peut maintenant le dire sans crainte : l'écriture arcanienne est féministe. À présent, peu de chercheurs en littérature en doutent ${ }^{2}$. Quoiqu'Arcan ait une image ambiguë sur le plan médiatique ou qu'elle fasse partie d'une génération d'auteur·e·s qui apparait parfois comme n'étant pas aussi concernée par les questions féministes que celles d'autres générations ${ }^{3}$, il est important de ne pas oublier que son écriture se classe bel et bien dans la catégorie des littératures qui interrogent les inégalités entre les hommes et les femmes ${ }^{4}$. Ainsi, on ne peut pas nier le fait qu'Arcan intéresse la critique littéraire : ses romans sont capitaux pour la littérature québécoise de l'extrême contemporain et pour les études sur le genre sexuel puisqu'ils critiquent le rôle des femmes dans la société occidentale contemporaine, dénoncent les stéréotypes masculins et féminins encore figés dans la société d'aujourd'hui et remettent en question les normes de genre sexuel $^{5}$, même s'ils vont inévitablement, comme le note Barbara Havercroft, reconduire certains stéréotypes ${ }^{6}$. Il n'est donc pas erroné de dire qu'à l'aide de son écriture - qui s'élabore selon différents procédés soigneusement choisis -, Arcan fait, entre autres, la critique d'une société contemporaine de surconsommation et d'hypersexualisation, où la domination masculine agit toujours sournoisement sur les rapports sexuels entre les femmes et les hommes.

On reconnait Arcan pour différents aspects : son image médiatique, son succès en France, son écriture autobiographique, son suicide ou ses propos crus et véridiques sur le pouvoir sexuel, le rapport au corps féminin, la chirurgie plastique et la pornographie, etc. Cela dit, on doit surtout la reconnaitre et l'admirer pour un élément des plus importants pour la littérature, c'est-à-dire la force de son écriture - une écriture qui, admettons-le, est d'une poétique magistrale. Métaphores, personnifications, répétitions, descriptions, figures d'amplification, d'opposition ou de substitution, Arcan les manie toutes d'une façon exemplaire. D'ailleurs, grâce à son écriture, cette auteure tente de désérotiser notre vision du membre viril et, par le fait même, dénonce certaines pratiques sexuelles phallocentriques qu'elle représente de façon négative, dans le but probable d'interroger le rapport entre le soi et l'autre dans la sexualité. En effet, son écriture n'est en aucun cas érotique, comme Arcan l'affirme lors de son entretien avec Guy A. Lepage à Tout le monde en parle à l'automne 2007 : «Je suis dans une écriture très analytique et très décortiquante, c'est-à- 
dire que $[\ldots]$ je désérotise la sexualité. Et j'en parle tout le temps : il est toujours question de cul dans mes livres, mais je le décortique. Donc ça le désérotise [...], je ne vais justement pas dans le sens du bandant quand j'écris $[\ldots] \gg^{7}$. Ce qui m'amène à avancer que, sur la question de la désérotisation chez Arcan, nous pouvons définir au moins trois grandes caractéristiques spécifiques à son écriture.

Premièrement, Arcan «multipli[e] les scènes graphiques ou les détails sexuels » dans le but de créer une «saturation », comme le note Cécile Hanania (123). Pour ce faire, elle utilise la description afin de décortiquer pour mieux désérotiser. On retrouve alors dans ses œuvres de grandes phrases et de longs comptes rendus d'événements sexuels. Par exemple, dans Folle, la narratrice Nelly décrit l'acte sexuel abusif de son ancien amant qui tente de l'initier à la pornographie (103-105). Il la pénètre en regardant des vidéos pornographiques, et ce, jusqu'à la jouissance. Pendant la pénétration, Nelly est en larmes, mais il ne s'en aperçoit qu'après avoir joui. Au lieu de représenter une scène d'échange sexuel, Arcan semble plutôt décrire celle d'une violence sexuelle, car plusieurs signaux ont été présentés à l'amant pour lui indiquer que sa partenaire n'était pas totalement favorable à la relation sexuelle. Conséquemment, on voit dans Folle que l'amant a un comportement machiste qui l'amène à ne pas respecter les femmes sur le plan de la pratique sexuelle et que la façon dont cet amant pense les femmes comme étant des objets sexuels établit facilement des manières d'agir qui peuvent dériver vers l'agression sexuelle. Par rapport à cette façon de traiter les femmes comme étant des êtres sans valeur, Arcan émet l'idée qu'entre objectification sexuelle et comportement sexuel problématique, il n'y a parfois qu'un pas.

Deuxièmement, Arcan conçoit une écriture anti-érotique. En utilisant des images choquantes qui ne sont aucunement sensuelles et qui viennent se juxtaposer à des passages sexuels, elle fait en sorte que la scène évoquée, plutôt que de stimuler le désir sexuel, suscite quelques interrogations, comme dans l'extrait du tableau ci-dessous où Charles, le personnage principal dans À ciel ouvert, se masturbe en regardant les seins refaits de sa maîtresse, Julie. Le roman relate l'histoire d'un triangle amoureux dont les trois membres (Charles Nadeau, Julie O'Brien et Rose Dubois) entrent dans une relation de jeux de pouvoir. D'abord ennemies redoutables, Julie et Rose se disputent l'amour de Charles et deviennent soumises à ses désirs - en usant de la chirurgie plastique jusqu'à pratiquer le fétichisme. De fait, Charles a des penchants sexuels singuliers parce qu'il aime les cicatrices sur les corps retouchés : il a un «désir cuisant » pour les prothèses mammaires, l'enflure des lèvres gonflées, les implants, les substances injectées, le durcissement, 
bref toutes « ces injures de la beauté enfoncée dans le corps [...]» (118). Dans l'extrait cité dans le tableau ci-dessous, Arcan représente Charles qui se masturbe devant le corps inerte de Julie et sous le regard étonné de Rose qui les surprend en flagrant délit. Le procédé littéraire utilisé pour rendre la scène anti-érotique est celui de l'antithèse, l' « inconscience » s'opposant à la « vivacité » (136), créant ainsi une opposition entre deux images qui font ressortir le déséquilibre entre l'objet et le sujet de l'action. «Vautrée sur le dos », Julie est « belle » et rappelle l'inertie d'une morte, ce qui contraste avec le geste animé de Charles, qui se masturbe avidement devant son fétiche. Cette scène, qui représente un objet sexuel passif à l'extrême, suscite davantage le questionnement que le désir sexuel parce qu'elle rappelle la domination masculine et révèle une critique féministe d'une certaine forme de pratique sexuelle phallocentrique - plus précisément une pratique où le membre viril de l'homme prend une place centrale au point où la femme ne devient qu'un être inanimé servant le plaisir de ce premier.

Troisièmement, Arcan désérotise la sexualité grâce à la mise en chantier d'un effet de profusion dans l'écriture qui s'apparente à la société de surconsommation dans laquelle sont pris ses personnages. Répétitions, énumérations, gradations, accumulations, c'est souvent dans l'amplification que se produit, dans l'écriture d'Arcan, une impression d'abondance qui banalise la sexualité tout en donnant l'illusion d'une accumulation mécanique éloignée de l'érotisme et de la sensualité, car plutôt rapprochée du pornographique ${ }^{8}$. Ce type de procédés est lié à la société moderne ${ }^{9}$ où l'être humain devient comparable à une marchandise que l'on dépersonnalise et que l'on traite comme un objet de consommation. À titre d'exemple de cet effet d'accumulation, j'ai cité dans le tableau ci-dessous l'extrait où, « entre leurs paroles » (135), Rose entrevoit différentes parties (queue, gland, seins, mamelons, langue) des corps sexués de Charles et de Julie. Ce type de procédé d'énumération d'images reliées à des parties du corps sexuellement connotées produit un rythme très expéditif qui évoque un visuel synecdochique rappelant les films pornographiques commerciaux qui se développent de manière industrielle ainsi que leurs pratiques sexuelles fabriquées.

Or, ce genre de renvoi au style pornographique est souvent, chez Arcan, caricatural et grossi, c'est-à-dire qu'il met en relief une expression exagérée du pornographique au point où l'auteure semble le parodier. Par conséquent, les œuvres arcaniennes n'entrent pas dans la catégorie des écritures pornographiques. Cela tient en grande partie au fait que le pornographique doit être dénudé d'intention moqueuse et critique. Dans Le ravissement du style : sémiostylistique 
pour une réception physiologique de l'art verbal, Cécile Laborde, dans sa définition du style pornographique, affirme que « [1]a pornographie thématique ne fait pas le pornographique » (94). Autrement dit, pour susciter le désir sexuel, le pornographique doit détenir certaines caractéristiques spécifiques. Par rapport à la parodie, Laborde note ceci : «La parodie, la grivoiserie, les calembours et autres jeux de mots burlesques ou douteux, annulent immanquablement l'effet pornographique. Le pornographique n'est pas du côté de la légèreté, ni de la frivolité, encore moins du jeu » (86). Puisqu'Arcan caricature le style pornographique de façon sardonique, on peut dire que son œuvre n'a pas comme but de susciter le désir sexuel, mais plutôt de critiquer certaines pratiques sexuelles. De fait, « [1] » écrire pornographique est un écrire de la distance » (107), c'est-à-dire que le texte doit maintenir un effet réaliste et un discours sexuel sans connotation péjorative, pour parvenir à susciter l'excitation. Chez Arcan, on dénote une tonalité ironique et un humour noir qui ne laissent pas entrevoir cette distance nécessaire à la création de désirs érotiques.

\begin{tabular}{|c|c|c|}
\hline \multicolumn{3}{|c|}{ L’écriture arcanienne et la désérotisation } \\
\hline Traits de l'écriture : & Procédés stylistiques : & Exemples : \\
\hline $\begin{array}{l}\text { La multiplication des } \\
\text { scènes graphiques et } \\
\text { des détails sexuels }\end{array}$ & $\begin{array}{l}\text { Descriptions } \\
\text { Grandes phrases, longs } \\
\text { comptes rendus } \\
\text { d'événements sexuels } \\
\text { abjects. }\end{array}$ & $\begin{array}{l}\text { «Après avoir regardé les photos, on a regardé les } \\
\text { séquences vidéo }[\ldots] \text {. Là tu as baissé mon } \\
\text { pantalon pour tirer ma petite culotte sur le côté et } \\
\text { me prendre par-derrière }[\ldots] \text {. Au même moment } \\
\text { tu as joui et je suis restée accroupie }[\ldots] » \text { (Folle, } \\
\text { 103-106). }\end{array}$ \\
\hline L'anti-érotique & $\begin{array}{l}\text { Figures d'opposition } \\
\text { Images choquantes qui } \\
\text { ne sont pas érotiques et } \\
\text { qui viennent se } \\
\text { juxtaposer à des } \\
\text { passages sexuels, } \\
\text { antithèse. }\end{array}$ & $\begin{array}{l}\text { «Julie était offerte les yeux fermés, vautrée sur le } \\
\text { dos, le T-shirt relevé au-dessus des seins, comme } \\
\text { assommée, belle dans son inconscience, tandis que } \\
\text { Charles se masturbait avec une vivacité que Rose } \\
\text { ne lui avait connue qu'à leurs propres débuts } \\
{[\ldots] \text { (A. ciel ouvert, 136). }}\end{array}$ \\
\hline $\begin{array}{l}\text { L'effet de profusion } \\
\text { (un rappel de la } \\
\text { société de } \\
\text { consommation ; l'être } \\
\text { humain comme une } \\
\text { marchandise) }\end{array}$ & $\begin{array}{l}\text { Figures d'amplification } \\
\text { Répétitions, } \\
\text { énumérations, } \\
\text { gradations ou } \\
\text { accumulations. }\end{array}$ & $\begin{array}{l}\text { «[...] entre leurs paroles elle entrevoyait la queue } \\
\text { de Charles, son gland, des seins surmontés de } \\
\text { mamelons durs, une langue }[\ldots] »(\hat{A} \text { ciel ouvert, } \\
\text { 135). }\end{array}$ \\
\hline
\end{tabular}

\section{Le sexe désérotisé : analyse du substantif « queue »}

Parmi tous ces procédés d'écriture qui participent à la désérotisation chez Arcan, on retrouve une insistance prééminente sur le substantif « queue » qui revient à plusieurs reprises de 
façon emphatique dans la plupart de son œuvre. D’abord, dans Putain, il renvoie au monde de la prostitution dominé majoritairement par les phallocrates désignés dans le roman et, ensuite, dans Folle, il est utilisé à titre de référent pour son amant - « homme dont le narcissisme le mène à se vanter des photos qu'il a prises de son propre pénis » (Smart 390) - et, enfin, dans À ciel ouvert, il finit par s'interchanger avec le substantif «sexe» et par connoter davantage sa vulgarité. Le substantif «queue » est donc employé dans les textes d'Arcan pour signifier différentes idées contre le phallocentrisme et les pratiques sexuelles phallocentriques.

En premier lieu, Putain est l'histoire d'une prostituée qui relate comment se sont enchaînées ses multiples expériences sexuelles et comment elle en est arrivée à discuter de cellesci avec un psychiatre. Dans ce premier roman, Arcan « lèv[e] simultanément le voile sur le système prostitutionnel ainsi que sur sa pierre angulaire, la marchandisation du corps féminin », comme l'écrit Anne Brown (63). Pour arriver à critiquer cette marchandisation du corps féminin par les personnages phallocrates mis en relief dans le roman, Arcan fait des rapprochements entre les hommes et leur membre viril. Par exemple, elle juxtapose « homme » et « queue » afin de suggérer que ceux-ci se correspondent, plus précisément que, dans le domaine de la prostitution, dire « homme » ou dire «queue » équivaut à la même idée référentielle pour la prostituée. Prenons l'extrait suivant à titre d'exemple :

Oui, la vie m'a traversée, je n'ai pas rêvé, ces hommes, des milliers, dans mon lit, dans ma bouche [...], j'ai tout vu et ça continue encore, tous les jours ou presque, des bouts d'homme, leur queue seulement, des bouts de queue qui s'émeuvent pour je ne sais quoi, car ce n'est pas de moi qu'ils bandent [...], c'est de ma putasserie, du fait que je suis là pour ça, les sucer, les sucer encore, ces queues qui s'enfilent les unes aux autres comme si j'allais les vider sans retour [...] (Putain, 19 ; je souligne).

La synecdoque «des bouts d'homme » prend la partie pour le tout. C'est aussi une métonymie particulière pour laquelle la relation entre le terme donné (des bouts d'homme) et le terme évoqué (queue) constitue une dépendance matérielle ou conceptuelle. Cet extrait met en évidence le rapport étroit qui existe entre l'homme et la femme dans le domaine de la prostitution féminine, un rapport qui n'est pas humanisant, mais strictement sexuel : l'homme n'est qu'un corps et il s'efface sous son membre, car, en ce qui concerne le rapport humain, il n'y en a pas. Lorsque la narratrice dit « des hommes dans ma bouche », on se figure facilement qu'elle veut en fait dire que ce sont « des queues » qui sont dans sa bouche ; quand elle personnifie les queues en disant qu'elles « s'émeuvent », on se doute également que ce sont en fait les hommes qui s'émeuvent; et, quand 
elle mentionne « ces queues qui s'enfilent », on sait qu'elle se réfère aux clients qui se succèdent. On fait face dans cet extrait à une masse d'hommes sans identité individuelle. En usant de cette figure de style qui substitue le mot «homme » au mot «queue » et inversement, Arcan semble parallèlement faire un rapprochement avec le système de pensée phallocrate : l'homme qui, avec son phallus, domine et déshumanise la femme en la traitant comme une espèce inférieure et en l'objectifiant sur le plan de la pratique sexuelle : «[c]e n'est pas de moi qu'ils bandent [...], c'est de ma putasserie » (19). L'auteure donne à sa narratrice une position dans le langage qui lui permet de réprouver la situation des femmes dans ce système : des femmes sans «moi », sans identité, déshumanisées. En ne désignant pas d'identité réelle aux hommes dans l'extrait cité plus haut, la narratrice Nelly, d'une certaine manière, combat le feu par le feu en les déshumanisant à son tour ; elle met en relief que, dans cette industrie du sexe, ceux-ci ne se résument qu'à leur membre viril, et parfois rien d'autre. Elle critique la déshumanisation par la dépersonnalisation : la narratrice fait voir que, dans cette façon qu'ont les hommes de traiter les femmes comme des objets sexuels, ils ont peu d'humanité et ils se dépersonnalisent.

D’autres figures de style sont utilisées de façon similaire dans Putain et il suffit de jeter un coup d'œil au tableau ci-dessous pour voir comment l'auteure exploite certaines stratégies d'écriture pour mettre en parallèle les hommes et leur membre viril. Le premier exemple est celui de la métaphore de la «queue » qui symbolise le commerce de la prostitution. En disant qu'elle se trouve prise, qu'elle bute sur la répétition d'un membre viril, la narratrice Nelly veut parler du fait qu'elle est contrainte au commerce de la prostitution. Les deux autres exemples, celui de la personnification «des queues » ou celui de la répétition du substantif «queue », se rapportent également au lien étroit entre « homme » et « queue » dans le système prostitutionnel phallocrate. En somme, par rapport à cette image de «queue dressée », plusieurs significations découlent de cette métaphore : une queue dressée dans la mesure où elle s'oppose à Nelly, une queue dressée pour ne pas dire «bandée », une queue dressée comme à l'écoute, disciplinée. De même, cette idée de «queue dressée » s'apparente certainement à celle du «phallus » afin de représenter le marché de la prostitution que condamne Arcan et qui est dominé par les phallocrates. Dans ce marché, selon le point de vue émis dans le roman, les hommes se compareraient aux bêtes et on apprendrait à les «dresser » à leur image. De surcroit, d'après le dictionnaire Larousse, «dresser » signifie «rendre docile un animal, l'habituer à des comportements qu'on exige de lui » ${ }^{10}$. Dans le domaine de la prostitution féminine, selon l'analogie de la narratrice, on attend uniquement des hommes 
qu'ils bandent bêtement et qu'ils paient. L'image de la « queue dressée », qui est au singulier et accompagnée de l'article indéfini «une», vient encore une fois, par la généralisation, dépersonnaliser les hommes et leur enlever leur individualité.

\section{L'emploi emphatique du mot « queue » dans Putain}

\begin{tabular}{|c|c|}
\hline Extraits : & Analyse littéraire : \\
\hline $\begin{array}{l}\text { «[...] il a suffi d'une seule fois pour me trouver } \\
\text { prise dans la répétition d'une queue dressée sur } \\
\text { laquelle je bute encore }[\ldots] »(\text { Putain, 22). }\end{array}$ & $\begin{array}{l}\text { Métaphore } \\
\text { Trouver prise, buter sur la répétition d'une queue = } \\
\text { prise dans le commerce de la prostitution. }\end{array}$ \\
\hline $\begin{array}{l}\text { «Il vaut mieux retourner à l'essentiel, au trafic de } \\
\text { ma bouche avec d'innombrables queues } \\
\text { impatientes de décharger et de recommencer à } \\
\text { s'impatienter [...]» (Putain, } 47) \text {. }\end{array}$ & $\begin{array}{l}\text { Personnification } \\
\text { Impatientes = caractéristique humaine (toujours } \\
\text { cette idée de la queue métonymie de l'homme). }\end{array}$ \\
\hline $\begin{array}{l}\text { «[...] mais je ne sais pas pardonner, je ne sais que } \\
\text { serrer les dents toujours plus sur l'insistance des } \\
\text { queues dans ma bouche, sur la queue de mon père } \\
\text { qui commerce avec les putains, et pas avec une } \\
\text { seule je suppose, car la putain en désigne } \\
\text { automatiquement une autre avec son corps qui par } \\
\text { nature en représente un autre, et ainsi se renvoient- } \\
\text { elles la queue de leurs clients, la queue de mon } \\
\text { père qui bande pour toute autre femme que la } \\
\text { sienne » (Putain, } 85) \text {. }\end{array}$ & $\begin{array}{l}\text { Répétition } \\
\text { «la queue } »=\langle\text { des queues » } \\
\text { La queue des clients (des queues) renvoie à la } \\
\text { queue de son père (la queue) comme la putain } \\
\text { renvoie à n'importe quelle autre putain. }\end{array}$ \\
\hline
\end{tabular}

Quant au roman Folle, celui-ci met en scène une femme en peine d'amour d'un homme qui l'a humiliée et l'a méprisée, comme l'euphémisme suivant le suggère : «Tu m'aimais en colon » (Folle, 176). Afin de montrer que certaines amours peuvent se dérouler de façon opposée à nos attentes et finir de manière cruelle, la narratrice Nelly raconte l'histoire de sa relation avec son ancien amant, journaliste pigiste français, installé à Montréal depuis quelques années déjà. Elle explique comment leur courte idylle a vite été suivie d'une rupture difficile et de sentiments dévastateurs, car, comme l'écrit Patricia Smart, cette histoire d'amour l'a réduite «à l'abjection » (388) : «Cherchant l'amour et une vie de couple normale [...], [Nelly] entre en relation avec un homme qui la traite de putain, crache sur elle et la renvoie chez elle quand elle lui déplaît » (388390). Dans le but de nous faire réfléchir sur certaines pratiques sexuelles exercées par le personnage de l'amant dans le roman ainsi que sur la vision qu'il a de son membre viril, Arcan met au point un procédé littéraire ingénieux : celui de la mise en emphase par le biais de la répétition du syntagme «ta queue » et c'est, entre autres, grâce à ce procédé qu'Arcan désérotise la sexualité. Comme on peut le voir dans les deux extraits qui figurent dans le tableau ci-dessous, le membre viril de l'ancien amant a été le centre d'intérêt tout au long de la relation entre les deux 
partenaires - surtout de son intérêt à lui. L'effet de la répétition du syntagme « ta queue » consiste à mettre l'accent sur l'importance qu'il y accordait. Combiné au déterminant possessif « ta », qui a plutôt une fonction accusatrice, le substantif «queue » est répété pour signifier à quel point la narratrice trouve abusive la place que ce membre viril détenait dans leur relation. Cette répétition $\mathrm{du}$ « ta queue » contribue alors à créer une saturation pour désérotiser le membre viril de son ancien amant. Le sens est plus ou moins évident : la «queue » représente le narcissisme phallocrate dont l'ancien amant était fervent et qui est dénoncé par la narratrice avec raillerie.

\begin{tabular}{|l|l|}
\hline \multicolumn{2}{|c|}{ La répétition du syntagme « ta queue » dans Folle } \\
\hline Extrait 1 : & Extrait 2 : \\
\hline «Tu aimais beaucoup ta queue et, souvent, tu la & «Tu as dit des choses qui ne sont pas faites pour \\
photographiais. Pour moi, c'était un geste de & être dites, que tu prenais ta queue en photo pour en \\
victoire, une forme de conquête, c'était le drapeau & faire une collection qui te permettait de la détailler, \\
américain hissé sur la Lune ; quand tu étais jeune, & que ta collection formait un panorama où les angles \\
tu as dû être un premier de classe. Pour ta queue, & les plus inaccessibles de ta queue se montraient en \\
j'ai inventé des formules qui te faisaient rire, je te & face sur ton écran, que de toutes les images [...] tu \\
soufflais à l'oreille ta queue pilier de notre amour & préférais encore celles de ta queue parce qu'elles \\
et ta queue trésor caché au fond du lit; ta queue & bandaient avec toi dans un jeu de symétrie. Tu as \\
que peu de femmes ont su apprécier à sa juste & dit que la grosseur de ta queue variait selon les \\
valeur me disais-tu souvent, ta queue que tu & jours et que c'était une question de force \\
questionnais parfois parce que tu ne savais pas où & d'excitation, que de saisir ta queue au dernier stade \\
la situer sur l'échelle des queues du monde où & de l'excitation avec l'appareil photo posait un \\
s'affrontaient les grandes races en ordre & problème parce que dès que tu la lâchais, elle \\
décroissant, [...] tu trouvais ma chatte étroite et & tombait, et qu'au moment où ta queue touchait sa \\
c'était pour ta queue un bon signe » (Folle, 27-28). & taille maximale, les secondes t'étaient comptées, ça \\
te rendait précoce. [...] [T]oi et moi avions compris \\
que le repli sur ta queue en disait long sur notre \\
avenir » (Folle, 96-97).
\end{tabular}

Si l'on retient cette hypothèse, on pourrait affirmer l'idée que de certains de ces passages dans Folle découle un humour noir, puisqu'ils dépeignent l'illogisme des propos et des pensées de l'ancien amant : en rapportant les discours tenus par cet homme, Nelly fait ressortir leur absurdité. Dans ce roman, l'humour noir se présente souvent sous des figures d'amplification et d'insistance, qui sont la marque même de l'écriture arcanienne. Considérant les aspects hyperboliques et caricaturaux de son style littéraire, il apparait que chez Arcan, comme mentionné plus haut, les faits sont souvent représentés de façon exagérée et grossie, ce qui témoigne d'une certaine forme de dérision typique de l'auteure. En guise de précision, on peut prendre l'extrait où Nelly fait de la « queue » de son ancien amant une source évidente de moquerie : «Tu aimais beaucoup ta queue et, souvent, tu la photographiais. Pour moi, c'était un geste de victoire, une forme de conquête, 
c'était le drapeau américain hissé sur la Lune ; quand tu étais jeune, tu as dû être un premier de classe » (27). On observe de l'humour noir en raison de la présence de l'hyperbole causée par la gradation ascendante. À partir du moment où photographier son membre viril devient un geste de conquête, on devine le phallocentrisme évoqué dans la métaphore du drapeau américain : rien de plus viril, de plus phallocrate, que de s'approprier une terre par le biais d'un drapeau. Quoi qu'il en soit, sous cet humour noir se trouve une tonalité ironique, en ceci que la narratrice semble vouloir montrer qu'elle dit le contraire de ce qu'elle pense. On pourrait alors aussi postuler l'idée qu'Arcan se range du côté des écrivaines « ironistes » dont parle Lucie Joubert dans Le carquois de velours. Concernant l'ironie au féminin, Joubert affirme que « rares sont les auteures qui vont ironiser bien précisément sur le membre viril, signe distinctif biologique de celui qui occupe les postes de commande » (135). Elle se demande si cela est fait par «pudeur » et « embarras » (135). À cet égard, Nelly met en relief l'idée qu'elle n'en a plus (d'embarras) : « c'est peut-être parce que l'embarras ne m'est plus accessible qu'il m'est possible d'écrire toutes ces choses » (Arcan, Folle, 92). C'est peut-être aussi la raison pour laquelle, contrairement aux autres ironistes étudiées par Joubert, Arcan, par le recours de sa narratrice Nelly, se moque à plusieurs reprises du membre viril.

Pour terminer, dans À ciel ouvert, on se rend compte de la vulgarité du substantif « queue ». Les extraits cités dans les tableaux plus hauts suggèrent l'idée que le choix de ce substantif n'est pas fait au hasard : dans Putain et dans Folle, Arcan, pour parler du membre viril, ne choisit pas d'utiliser le substantif «pénis », qui fait partie du registre standard, et elle ne choisit pas non plus d'utiliser le substantif « sexe », qui, lui, tient plutôt du registre soutenu ; elle choisit d'utiliser « queue ». Ce choix terminologique vient amplifier la représentation négative du membre viril, car son aspect familier est de nature crue et vulgaire, rappelant celle des hommes dépersonnalisés dans l'industrie de la prostitution et celle de l'ancien amant. De cette manière, le substantif « queue »a des propriétés pornographiques qui ont pour fonction, chez Arcan, de désérotiser la sexualité. On s'en rend compte étant donné que, dans À ciel ouvert, Arcan vacille entre l'utilisation des substantifs «sexe » et «queue » pour parler du membre viril du personnage principal Charles, comme les extraits cités dans le tableau ci-dessous le mettent en lumière. 


\begin{tabular}{|c|c|}
\hline \multicolumn{2}{|c|}{ Vacillement entre les substantifs « sexe » et « queue » dans À ciel ouvert } \\
\hline Le sexe : & La queue : \\
\hline $\begin{array}{l}\text { "Curieusement le sexe de Charles semblait ne pas } \\
\text { être au rendez-vous, peut-être parce que Charles } \\
\text { n'avait pas laissé Julie le toucher, peut-être parce } \\
\text { qu'il ne bandait pas » (À ciel ouvert, 101). }\end{array}$ & $\begin{array}{l}\text { "Mais Charles souffrait toujours de son désir } \\
\text { cuisant, de sa queue dure à en avoir mal }[\ldots] »(\hat{A} \\
\text { ciel ouvert, 118). }\end{array}$ \\
\hline $\begin{array}{l}\text { «le sexe de Charles qui en avait joui aussitôt, en } \\
\text { deux grands jets de sperme dirigés vers ses seins } \\
{[\ldots] »(160) \text {. }}\end{array}$ & $\begin{array}{l}\text { «[Rose] les voyait parler, fumer et boire, mais entre } \\
\text { leurs paroles elle entrevoyait la queue de Charles, } \\
\text { son gland, des seins surmontés de mamelons durs } \\
{[\ldots] »(135) \text {. }}\end{array}$ \\
\hline $\begin{array}{l}\text { "Charles regardait toujours son écran, la main sur } \\
\text { son sexe, mais il ne voyait plus les images » (204). }\end{array}$ & $\begin{array}{l}\text { "Charles s'était assis sur le divan brun en se } \\
\text { prenant la tête dans les mains. Sa queue pendant } \\
\text { comme une langue molle }[\ldots] »(211) \text {. }\end{array}$ \\
\hline
\end{tabular}

Deux conséquences ressortent de ce vacillement. De l'une, on prend conscience de l'importance du choix du substantif « queue » dans Putain et dans Folle. De l'autre, on se demande si Charles n'a pas un statut particulier par rapport aux autres hommes représentés dans les romans précédents. En effet, le fait d'utiliser le substantif «sexe » plutôt que celui de «queue » est-il une tentative d'érotisation ou de neutralité du langage, car il semble qu'en lisant «le sexe de Charles » on est plus portés à être cléments devant l'histoire du personnage masculin et à comprendre sa situation et ses problèmes au lieu d'en être choqués ou irrités ? Comme on l'a vu plus haut, Charles a une sexualité déviante. Par contre, cette déviance est due, entre autres, à des problèmes survenus pendant son enfance ${ }^{11}$. Au cours de la lecture, on est tentés de prendre son parti lorsqu'on le voit peu à peu périr de ses penchants sexuels. Honteux de ceux-ci, il finit d'ailleurs par se donner la mort. Écrire « le sexe » à la place de «la queue» change le registre du discours et ce nouveau registre semble ne pas attaquer l'identité masculine de Charles de la même façon que celles des autres hommes dans l'univers arcanien. Ce vacillement entre « sexe » et «queue » est aussi la cause d'une écriture en mouvement - une écriture qui, contrairement à celle retrouvée dans Putain et dans Folle, ne critique pas aussi fortement l'identité masculine des personnages - et d'une posture d'écrivaine moins portée vers la subjectivité présente dans l'autofiction. Se montrant plus nuancée à l'égard de son personnage Charles et de ses pratiques sexuelles différentes, Arcan illustre le fait que, dans son univers littéraire, ce dernier est lui aussi victime d'un système. Arcan parait mettre en évidence que cette société dépeinte dans son œuvre - celle de surconsommation et d'hypersexualisation découlant du patriarcat - a également des répercussions négatives sur les hommes qui, de leur côté, peuvent parfois souffrir du pouvoir qui leur a été accordé. La domination 
masculine présente dans la société représentée n'a pas des conséquences seulement sur les femmes, mais assurément sur certains hommes, surtout lorsque certains d'entre eux ne correspondent pas aux modèles dominants de la masculinité ou, comme Charles, ont des goûts sexuels qui diffèrent largement des normes sociales. À ciel ouvert témoigne de nouvelles nuances présentes dans l'écriture arcanienne : si, avec cette œuvre, Arcan critique encore la société phallocentrique, elle ne rejette cependant pas ici l'idée que les femmes ne sont pas les seules victimes de la domination masculine.

La représentation des pratiques sexuelles chez Arcan interroge la place du membre viril comme objet de pouvoir dans la société occidentale. Je me suis intéressée à la sexualité «désérotisée » chez Arcan pour me rendre compte qu'en allant à l'inverse de l'érotisme et en remettant en question certaines postures, l'auteure critique un type de pratiques sexuelles en particulier. Pour s'opposer à la domination masculine dans le champ de la sexualité, elle désérotise des formes de pratiques sexuelles machistes et phallocentriques. Elle décortique ainsi les scènes sexuelles au moyen d'un style anti-érotique et d'un effet de profusion dans l'écriture, et elle en sature ses textes, dans le but de nous faire comprendre que notre société contemporaine souffre d'un bagage historique phallocentrique très fort. Elle examine aussi des pratiques sexuelles où le rapport humain (de l'un·e avec l'autre) n'est pas toujours pris en considération. À l'heure où la question des violences sexuelles est criante d'actualité et où on dénonce de plus en plus les pratiques sexuelles misogynes, il m'apparait crucial de revisiter les œuvres d'Arcan : celles-ci, en dénonçant l'importance accordée au membre viril dans les rapports sexuels entre les hommes et les femmes, attirent l'attention sur certaines agressions sexuelles provoquées par l'objectification féminine et la domination masculine. Par rapport à la question des pratiques sexuelles, le respect de soi et de l'autre est crucial, et c'est ce qu'exposent les œuvres d'Arcan : interrogeons nos pratiques pour nous diriger vers une sexualité plus égalitaire, pour l'un·e et pour l'autre.

\section{Bibliographie}

Arcan, Nelly. À ciel ouvert. Paris : Seuil, 2007.

---. Folle. Paris : Seuil, 2004.

---. Putain. Paris : Seuil, 2001.

Bauman, Zygmunt. La vie liquide. Paris : Pluriel, [2005] 2013.

Beauvoir de, Simone. Le deuxième sexe, Tome 2. Paris : Gallimard, 1949. 
Boisclair, Isabelle, Christina Chung, Joëlle Papillon et Karine Rosso, dir. Nelly Arcan. Trajectoires fulgurantes. Montréal : Remue-ménage, 2017.

Bourdieu, Pierre. La domination masculine. Paris : Seuil, 1998.

Brown, Anne. «Une lecture sociologique de Putain ou la démystification de la femme corpssexe ». Québec Studies 41 (2006) : 63-82.

Butler, Judith. Ces corps qui comptent : de la matérialité et des limites discursives $d u$ « sexe ». Tr. Charlotte Nordmann. Paris : Amsterdam, 2009.

Connell, Raewyn. Masculinités : enjeux sociaux de l'hégémonie. Tr. Meoïn Hagège et Arthur Vuattoux. Paris : Amsterdam, 2014.

Corbin, Alain. Histoire de la virilité. Tome 2. Le triomphe de la virilité. Le XIXe siècle. Paris : Seuil, 2011.

Courtine, Jean-Jacques, dir. Histoire de la virilité. Tome 3. La virilité en crise ? Paris : Seuil, 2011.

Courbet, David. Féminismes et pornographie. Paris : La Musardine, 2012.

Daniélou, Alain. Le phallus. France : Pardès, 1993.

Delvaux, Martine. «Écriture et nudité. Les femmes de Nelly Arcan et de Vanessa Beecroft ». Tangence 103 (2013) : 79-91.

Freud, Sigmund. Euvres complètes, tome XVII. Dir. André Bourguignon et Pierre Cotet. Tr. collectif. Paris : PUF, 1992.

Goux, Jean-Joseph. Renversements : l'or, le père, le phallus et le langage. Paris : Antoinette Fouque, 2009.

Hanania, Cécile. «Putain de Nelly Arcan. Texte écran et "je" de miroirs, une écriture de la dépersonnalisation ». Dalhousie French Studies 92 (2010) : 113-125.

Havercroft, Barbara. «(Un)tying the Knot of Patriarchy: Agency and Subjectivity in the Autobiographical Writings of France Théoret and Nelly Arcan ». Auto/biography in Canada : Critical Directions. Dir. Julie Rak. Waterloo : Wilfrid Laurier UP, 2005 : 207-234.

Joseph, Sandrina Objets de mépris, sujets de langage. Montréal : XYZ, 2009.

Joubert, Lucie. Le carquois de velours : l'ironie au féminin dans la littérature québécoise 19601980. Canada : L'Hexagone, 1998.

Laborde, Cécile. Le ravissement du style : sémiostylistique pour une réception physiologique de l'art verbal. Paris : Champion, 2016. 
Labrosse, Claudia. «L'impératif de la beauté du corps féminin : la minceur, l'obésité et la sexualité dans les romans de Lise Tremblay et Nelly Arcan ». Recherches féministes 23.2 (2010) : 209-213.

Scott, Joan W. De l'utilité du genre. Tr. Claude Servan-Schreiber. France : Fayard, 2012.

Smart, Patricia. De Marie de l'Incarnation à Nelly Arcan : se dire, se faire par l'écriture intime. Montréal : Boréal, 2014.

Larousse. https://www.larousse.fr/dictionnaires/francais.

Tabet, Simon. Le projet sociologique de Zygmunt Bauman : vers une approche critique de la postmodernité. Paris : L’Harmattan, 2014.

Vigarello, Georges, dir. Histoire de la virilité. Tome 1. L'invention de la virilité de l'Antiquité aux Lumières. Paris : Seuil, 2011.

\section{Notes}

${ }^{1}$ En ce qui concerne l'identité masculine, on parle maintenant beaucoup plus en termes de «masculinité » que de « virilité ». Ce changement de terminologie marque une rupture, comme le souligne Courtine : "Que "masculin" en soit venu à supplanter "viril" est bien le signe qu'il y a, décidément, quelque chose qui a changé dans l'empire du mâle » (8).

${ }^{2}$ Cela dit, certains critiquent peuvent penser le contraire. Par exemple, en 2010, Claudia Labrosse note l'ambiguïté du discours féministe d'Arcan. On le remarque dans l'extrait suivant de son article : «[...] Arcan tient un discours féministe des plus ambivalents. Dénonçant les stéréotypes qui encouragent la survivance de la femme patriarcale (la femme-objet), elle les reconduit simultanément en faisant évoluer des narratrices et personnages avides de se conformer aux normes extérieures » (26). Labrosse évoque également le fait que, au départ, «nombreuses sont les femmes qui hésitent à qualifier Arcan d'auteure féministe » (41).

${ }^{3}$ Barbara Havercroft avance des arguments similaires en 2005. Dans son article «(Un)tying the Knot of Patriarchy : Agency and Subjectivity in the Autobiographical Writings of France Théoret and Nelly Arcan », elle écrit qu'Arcan apparait comme faisant partie d'une « younger generation of women writers much less concerned with voicing an explicit feminist agenda » (208).

${ }^{4}$ Pensons notamment à son livre Putain qui, tel que l'affirme Cécile Hanania, «s'est vu amplement commenté par la critique académique comme un écrit féministe » (113). À ce propos, Hanania ne donne pas d'exemple de critiques, mais nous pouvons penser aux articles publiés dans Nelly Arcan. Trajectoires fulgurantes (2017), qui le montrent très bien.

${ }^{5}$ À ce sujet, Martine Delvaux écrit la réflexion suivante : «Il en est ainsi, aussi, de Nelly Arcan. Dans son imaginaire, les femmes ne sont pas des arbres mais des étoiles qui brillent dans le noir, "têtes à peau claire [qui forment] des points de repère dans l'obscurité du bar" (F, p. 22) et forment une constellation» (88). On sait que les étoiles sont une thématique présente dans quelques-uns des récits arcaniens - particulièrement dans Folle où les tarots, les étoiles, l'univers et les astres sont très imposants - et qu'elles peuvent, tel que l'affirme Delvaux, renvoyer facilement à l'image de la femme.

6 Voir l'article de Havercroft, intitulé «(Un)tying the Knot of Patriarchy : Agency and Subjectivity in the Autobiographical Writings of France Théoret and Nelly Arcan ».

${ }^{7}$ https://www.dailymotion.com/video/xalxbg

8 Bien qu'on sache qu'érotisme et pornographique sont liés de différentes façons et que «la pornographie d'aujourd'hui pourrait consister en l'érotisme de demain » (Courbet 16), il semble qu'il y ait un désir d'opposer l'érotisme au pornographique chez Arcan - une façon probablement de juger négativement l'industrie du sexe. Il faut dire aussi qu'opposer érotisme et pornographique est un lieu commun (Courbet 15) et que la définition de ce qu'est la « pornographie » est propice à changer selon les perspectives et les époques (Laborde 39). Par «pornographie », on fait ainsi surtout référence aux images animées sous la forme de vidéos ou de films grand public largement diffusés qui vont, la plupart du temps, être critiqués par les féministes à cause de la position qu'y détiennent les femmes. Dans 
cette logique, l'idée de l'érotisme et celle du pornographique, sans nécessairement être opposées, comporteraient des aspects différents dans la construction sociale de la réalité chez Arcan.

${ }^{9}$ J'utilise le terme « société moderne » pour faire référence à la pensée de Zygmunt Bauman, lorsque ce dernier parle de la «société moderne liquide» pour évoquer notre contemporanéité. À ce propos, Simon Tabet, dans Le projet sociologique de Zygmunt Bauman, affirme ceci : «Le terme "liquide" émerge dans la pensée de Bauman à la fin des années 1990 : sa première théorisation se trouve dans son ouvrage Work, Consumerism and the New Poor (1998). Il y définit alors la société liquide en miroir de ce qu'il nomme la société solide, à la manière de la postmodernité vis-àvis de la modernité : la société solide décrit une société de production visant à créer collectivement les cadres structurels de l'organisation commune, comprenant ainsi, par essence, une tendance totalitaire. À l'inverse, la société liquide désigne notre société de consommation dont l'idéal de liberté ne trouve plus de fondement sécurisant pour les individus, et dont la contre-utopie réside dans une fragmentation généralisée de la société » (67). On peut aussi lire son livre La vie liquide (2005) qui traite de la question de la société moderne.

${ }^{10} \mathrm{https}: / /$ www.larousse.fr/dictionnaires/francais/dresser/26787

${ }^{11}$ Dans À ciel ouvert, Julie essaie d'interpréter le comportement de Charles par des conversations où il parle de son enfance. « Ne [pouvant] plus s'arrêter de parler » (68), Charles lui explique les raisons qui font qu'il est devenu un photographe et qu'il se soucie du détail. Par analogie, on comprend alors que le portrait psychologique de ce personnage masculin (ses penchants sexuels et ses actions) est influencé par son enfance, plus spécifiquement par le métier de son père et par la manière dont ce dernier le traitait : "Charles avait gardé de son enfance des souvenirs terribles et remplis d'angoisse, justement, d'une vraie angoisse de pièces de viande suspendues, celles de son père et de sa boucherie; d'une chambrette surtout à l'intérieur de la boucherie chargée du froid et de l'odeur de la mort où son père avait l'habitude de l'enfermer chaque fois qu'il avait des crises d'angoisse et qu'il réclamait sa mère et ses sœurs parties vivre dans une autre ville, alors qu'il avait douze ans. Il lui avait parlé de ses visions de pièces de viande ouvertes, dépecées, cordées, de son sentiment que la vie allait prendre dans cette chair pour l'attraper, le mettre en pièces destinées à être à leur tour suspendues et, qui sait, à se remémorer cette vie où elles formaient un tout » (64). 